\title{
LA GUERRA DIGITAL POR LA INFORMACIÓn En ÁFRICA: CAPITALISMO INTERRACIONAL DE LA MANO DE GOOGLE, FACEBOOK, IBM Y MICROSOFT
}

\section{The Digital War for the Information in Africa: Global Capitalism in the Continent with Google, Facebook, IBM \& Microsoft}

\author{
Sebastián Ruiz-Cabrera \\ Universidad Loyola Andalucía / Grupo de Estudios Africanos (GEA) \\ E-mail: sebaprensa@gmail.com
}

0 Autor

(9)

\begin{abstract}
El clima de privatización y liberalización de los años ochenta y noventa en África allanó el camino para que algunos de los más importantes conglomerados de medios africanos sembraran las primeras semillas del plan corporativo transnacional. Al mismo tiempo, el tsunami liberalizador de esta época favoreció al actual panorama en el que las multinacionales se mueven sin marcos reguladores en el continente. Este es el caso de los gigantes de las telecomunicaciones quienes se encuentran instalados desde hace años en África previendo ese aforismo exponencial y cacareado desde los sectores liberales: "África crece. Y el futuro está alli". Un crecimiento, no obstante, sin desarrollo.

La narrativa dominante de empresas como Microsoft, IBM, Google o Facebook es que pretenden conectar a millones de personas, sin embargo, con el presente artículo trataremos de trazar otras vinculaciones, especialmente tras los escándalos de Facebook y Cambridge Analytica en procesos electorales, que nos ayudarán a entender esta dinámica que se insertaría en una guerra por el control de la información internacional y global. Un "conflicto bélico" que por uno de sus principales escenarios y laboratorios tiene al continente africano.
\end{abstract}

Filantropía; Internet; democracia; brecha digital.

Philanthropy; Internet; democracy; digital gap.

Key mords 
The climate of privatization and liberalization of the eighties and nineties in Africa paved the way for some of the most important conglomerates of African media to plant the first seeds of the transnational corporate plan. At the same time, the liberalizing tsunami of this time favored the current scenario in which multinationals move without regulatory frameworks in the continent. This is the case of the giants of telecommunications who have been installed for years in Africa foreseeing that exponential and vaunted aphorism from the liberal sectors: "Africa grows. And the future is there". A growth, however, without development.

The dominant narrative of companies like Microsoft, IBM, Google or Facebook is that they intend to connect millions of people, however, with this article we will try to draw other links, especially after the scandals of Facebook and Cambridge Analytica in electoral processes, which will help to understand this dynamic that would be inserted in a war for the control of international and global information. A "warlike conflict" that has one of its main scenarios and laboratories in the African continent.

\section{Introducción. Recetas neoliberales y juego sucio}

La conocida como "ola de las independencias africanas" en la década de 1960, con la consiguiente emancipación política y económica de sus antiguas metrópolis, se vería truncada unos años más tarde. Si bien la situación de inestabilidad que sufrieron muchos de estos nuevos Estados indudablemente frenó su desarrollo económico, la idea de que los problemas financieros son intrínsecamente internos es un error común. Tal perspectiva ignora el papel meridiano que las instituciones extranjeras -sean Estados u organismos internacionales (Ruiz-Giménez, 2012) - han jugado en el sabotaje de los caminos para un desarrollo soberano del conjunto de África (Oya \& Santamaría, 2007).

Mientras que algunas de las intervenciones occidentales inmediatamente posteriores a las independencias fueron flagrantes ${ }^{1}$ fue, no obstante, el cambio ideológico que barrió a Gran Bretaña y Estados Unidos hacia el fundamentalismo de mercado en la década de 1980 -traducido en el binomio Reagan-Thatcher- lo que sometió a la mayoría del África al sur del Sahara (ASS) a la hemorragia económica que duraría aproximadamente hasta el 2010.

Cuando la Crisis de la Deuda golpeó el mundo en 1982, el Fondo Monetario Internacional (FMI) y el Banco Mundial (BM) elaboraron estrictos planes de ajuste estructural (PAE) para los países del sur. En concreto, estos programas obligaron a 29 países del ASS a adoptar el modelo neoliberal antes de que terminara la década y este panorama impulsó la actual fotografía que podemos encontrar en el continente africano. Workneh lo resume de la siguiente manera:

El clima de privatización y liberalización de los años ochenta y noventa allanó el camino para que algunos de los más importantes conglomerados de medios africanos sembraran las primeras semillas del plan corporativo transnacional. (Workneh, 2017, p. 287)

1 Destacan algunos asesinatos relevantes: Patrice Lumumba, en 1961, Primer Ministro de la República Democrática de Congo (Estados Unidos desclasificó un informe de la CIA en septiembre de 2014 en el que se detallaba todo el plan para la "eliminación de Lumumba”. Más información aquí: http://bit.ly/2kd97eV); Sylvanus Olympo, en 1963, primer presidente de Togo; o Thomas Sankara, en 1987, el revolucionario que emprendió una reforma estructural en su país, Burkina Faso. 
Estos cambios estructurales tuvieron su onda expansiva en el mercado favoreciendo la creación de nuevas emisoras de radio internacionales en las ciudades africanas (Vittin, 2002), de nuevos canales de televisiones extranjeros vía satélite y por cable (Ba, 1999) y de miles de medios de comunicación y otras fuentes a través de Internet (Banda et al., 2009). El contenido en los medios locales también se transformó desde el culto de la personalidad y la propaganda estatal $^{2}$, hasta una multiplicidad de discursos críticos, diversos y políticamente comprometidos que reflejaban las preocupaciones populares o defendían los intereses de grupos particulares (Nyamnjoh, 2005) o aquellos que se centraban en informar de los hechos y a veces incluso en hacer un intento de periodismo de investigación (Frère, 2000, 2011).

La realidad actual es que, en apenas dos décadas, el continente ha pasado de una situación de escasez, donde cada país tenía algunas cabeceras financiadas por el Gobierno o por el partido único -y una única emisora nacional (radio y televisión)-, a una profusión impresionante de periódicos y estaciones que compiten por el mercado nacional o tienen ambiciones más allá de sus territorios (Tudesq, 2002; Ruiz-Cabrera, 2016).

Si tuviéramos que enumerar algunas de las características de la industria de los medios en el continente hoy por hoy serían: el aumento de la digitalización, una segmentación en la audiencia y los lectores, un programa confuso de apuesta por la transformación social ${ }^{3}$ y enormes desafíos tanto en la base de la profesión (formación) como en su aplicación (represión, censura, sobornos e instrumentalización).

Al mismo tiempo, el tsunami liberalizador de los ochenta favoreció al actual panorama en el que las multinacionales se mueven sin marcos reguladores en el continente. Este es el caso de los conglomerados como Google, Facebook, Microsoft o IBM quienes se encuentran instalados desde hace años en África previendo ese aforismo exponencial y cacareado desde muchos sectores: "África crece. Y el futuro está allî". Las razones de esta tendencia son en realidad bastante simples: África posee siete de las diez economías del mundo que más rápido crecen. Y estas empresas se encuentran frente a dos opciones que de forma especulativa les auguran beneficios desorbitados. No obstante, se trata de un crecimiento sin desarrollo, es decir, coyuntural y no estructural.

En primer lugar, este crecimiento económico ha favorecido la aparición de una clase social con poder adquisitivo, una dinámica que las compañías tecnológicas -aunque no solo ellastraducen meramente como cifras; es decir, como un nuevo mercado en el que los consumidores estarían hambrientos por sus productos (Melber, 2016). En segundo lugar, este nuevo paradigma aplicado al caso de ASS presenta una infraestructura de acceso a Internet o a las

2 Varios de los primeros presidentes africanos entendieron a los medios de comunicación como posibles vehículos para difundir sus ideologías políticas basadas en la unidad nacional después del periodo de colonización. Algunos ejemplos: el presidente Jomo Kenyatta (Kenia) instaba a la prensa para que siempre buscase la unidad nacional o "Harambee" en lugar del aislamiento; el presidente de Ghana Kwame Nkrumah reivindicaba el discurso de la "Personalidad africana"; lo mismo ocurría con el movimiento, más que discurso, de la "Negritud" en el Senegal de Leopold Senghor; el "Ujamaa" en Tanzania, de la mano de uno de los padres de los "socialismos africanos", Julius Nyerere; la narrativa del "Humanismo" en Zambia, respaldada por su primer presidente Kenneth Kaunda; o el "Chimurenga" (que quiere decir lucha revolucionaria) en el Zimbabue de Robert Mugabe.

3 Cabe destacar algunos ejemplos de países donde el uso de redes sociales como Twitter o Facebook, en cuanto canales de comunicación, sí han servido de catalizadores para encauzar el descontento social: Senegal o Burkina Faso en África Occidental; República Democrática de Congo o Nigeria en África Central; Zimbabue o Sudáfrica, en África Austral; o Etiopía, Ruanda y Kenia en África del Este. Para más información en español: Castel, A., \& Bajo, E. C. (2013). Redes sociales para el cambio en África. Madrid: Los Libros de la Catarata. Y en inglés: Branch, A., \& Mampilly, Z. C. (2015). Africa uprising: Popular protest and political change. London: Zed Books.

\section{Google, Facebook, Microsoft o IBM se encuentran instalados desde hace años en África}


redes móviles limitada y su plena adopción se encuentra todavía en una etapa muy incipiente en enormes zonas del continente. Es decir, como la instalación de redes de electricidad es compleja y cara, se opta por lo digital que abarata los costes y triplica los beneficios para empresas como Facebook o Google que enmascaran sus objetivos bajo un barniz de corte filantrópico (Ruiz-Cabrera, 2017).

Es por esta razón que las principales compañías están compitiendo por generar las condiciones propicias para alcanzar al mayor número de usuarios "conectados" posibles y que estos circulen por las vías de una información controlada. Se pone de manifiesto que la dinámica de la comunicación de masas queda superpuesta siempre a las relaciones de poder.

\section{Medios de comunicación africanos en el contexto internacional de la información}

Los grandes avances para la libertad de los medios en el ASS se produjeron bajo la etiqueta acuñada como la tercera ola de democratización ${ }^{4}$ que barrió las costas del continente. La Declaración de Windhoek de 1991 - una llamada a la libertad de los medios redactado por periodistas africanos, respaldado posteriormente por la UNESCO, y visto como un momento crucial para el desarrollo de los medios en la región-, llegó a Benín (1991), Malí (1992), Ghana (1992), Malaui (1994), Sudáfrica (1994) y a otros países que estaban abandonando a los Estados autoritarios y regímenes militares a favor de políticas más abiertas.

Estas transiciones no siempre fueron inmediatas o incluso exitosas. En muchos de estos casos, los titulares continuaron abusando de su poder para manipular los resultados electorales en los años venideros. Sin embargo, la mencionada Declaración de Windhoek marcó claramente el comienzo de más de una década de progreso constante en ASS hacia mayores derechos democráticos y libertades para los medios. Kwame Karikari, un destacado estudioso de los medios africanos y fundador del Media Foundation for West Africa, escribió años después sobre la transformación que había presenciado en la libertad de prensa durante esta ola de democratización:

El boom mediático de finales de la década de 1980 y principios de la década de 1990, que acompañó al movimiento por las reformas democráticas en África, transformó el panorama de los medios del continente de la noche a la mañana. Terminó con el control y el monopolio casi absoluto del Gobierno y marcó el comienzo de un vibrante pluralismo. De repente, las calles de las capitales de África estaban inundadas de periódicos. La "cultura del silencio", impuesta primero bajo el colonialismo y luego por las dictaduras militares poscoloniales y los Estados autocráticos de partido único, se rompió groseramente. (Karikari, 2010)

No obstante, más importante que el crecimiento en los periódicos privados e independientes en este periodo fue el crecimiento de las estaciones de radio y televisión privadas y comunitarias, que ocurrieron incluso en los Estados que previamente habían mantenido un estricto control de las ondas. El llamamiento de la Declaración de Windhoek para que los organismos de radiodifu-

4 El término "tercera ola" fue acuñado por Samuel Huntington; ver Huntington, S. P. (1993). The third wave: Democratization in the late twentieth century. London, Norman: Univ. of Oklahoma Pr.

Como la instalación de redes de electricidad es compleja y cara, se opta por lo digital que abarata los costes y triplica los beneficios 
sión estatales de la región se transformaran en emisoras públicas no tuvo éxito, pero al menos se permitió que floreciera la radiodifusión privada.

La diversificación del sector de los medios de comunicación estuvo acompañada de mejores marcos jurídicos e institucionales en apoyo de la libertad de los medios de comunicación. Las nuevas constituciones adoptadas en la región en las últimas tres décadas han consagrado algún derecho a la libertad de expresión, aunque muchas constituciones todavía conservan subcláusulas que imponen restricciones excesivas a ese derecho relacionadas no solo con la seguridad nacional, sino también con el orden público, la moralidad, los insultos u otras nociones vagas que han sido utilizadas para criminalizar el periodismo crítico.

Pero a medida que la democratización de ASS en la década de 1990 fue de la mano del progreso de la libertad de prensa, el inicio de una llamada recesión democrática tras el cambio de milenio revirtió algunos de los avances que se habían conseguido hasta el momento (Diamond, 2015). Entre 2009 y 2017, el promedio de libertad de prensa para los países del ASS 5 empeoró, pasando de 58,4 a 59,7 (donde 1 es la mejor puntación y 100 la peor).

$\mathrm{Al}$ igual que en otras partes del mundo, las amenazas a la libertad de prensa en África ya no se limitan exclusivamente a actos abiertos de intimidación o censura por parte del Estado, sino que ahora emanan de formas más sutiles en las que se rapta a los medios, y en las que las élites políticas, frecuentemente en connivencia con las oligarquías de estos países, usan una combinación de poder político y económico para regular y controlar las narrativas de los medios (Shiffrin, 2017). Si bien este rapto ha sido siempre una amenaza, los sistemas de medios ahora son más vulnerables que nunca a estas formas de manipulación, debido a la interrupción y el cambio en los modelos de negocio periodísticos aportados por las nuevas tecnologías digitales. En los países donde los medios se han alineado con los actores políticos, los periodistas a menudo se enfocan en asuntos que marginan a segmentos importantes de la población.

Mientras tanto, la esperanza inicial de que la comunicación digital interrumpiría la polarización política de los medios en ASS ha dejado paso lentamente a la preocupación por las nuevas amenazas al pluralismo informativo en el entorno de los medios digitales.

En Kenia, un país con uno de los sistemas de medios más vibrantes del continente, destaca la creciente preocupación por la tecnología digital. En las elecciones de 2007, un sistema de mensajes de teléfonos (SMS) proporcionó una plataforma para la difusión de rumores y desinformación que pudo haber contribuido a la violencia postelectoral (Abdi, 2008). Diez años después, las fake news en Internet (que aún sigue siendo una etiqueta difusa y, en ocasiones, contraproducente $^{6}$ ) han destacado como una preocupación importante: en la primera encuesta nacional realizada en Kenia sobre este asunto, el $90 \%$ de los 2.000 encuestados informó haber visto noticias falsas o deliberadamente engañosas relacionadas con las elecciones de $2017^{7}$.

Los medios digitales tampoco han demostrado ser tan autónomos a la influencia de las élites en el ASS como se esperaba. Camerún y Etiopía, por ejemplo, se mostraron dispuestos y capa-

5 Hay 49 países en el África subsahariana en el conjunto de datos de Freedom House. Sudán del Sur se agregó al conjunto de datos en 2012 después de su independencia de Sudán, y Somalilandia, aunque no fue reconocido anteriormente como país, se incluyó en el conjunto de datos en 2015. Este informe también incluye a Sudán como un país subsahariano, aunque no es siempre considerado parte de la región.

6 Para una mejor comprensión de la problemática bajo el concepto fake news véase Wardle, C. (2017). Fake News. It's Complicated. Recuperado de https://firstdraftnews.com/fakenews-complicated/.

7 Recuperado de https://portland-communications.com/publications/reality-fake-news-kenya/

Entre 2009 y 2017, el promedio de libertad de prensa para los países del África al Sur del Sahara empeoró 
ces de cerrar Internet o bloquear contenidos específicos de una forma que no se consideraba una amenaza hace unos pocos años (UNESCO, 2018). En este contexto, el pluralismo de los medios sigue siendo un ideal elusivo. Si bien se han logrado avances democráticos en algunos países del continente, las organizaciones de medios, los periodistas y los blogueros siguen bajo presión en muchos lugares.

\section{Tecnologías de la información y la comunicación (TIC): ¿al servicio de quién?}

Existe una extensa literatura sobre la naturaleza y los impactos del cambio tecnológico en el desarrollo socioeconómico (por ejemplo, Rogers, 1962; Ruttan, 2001; Nye, 2006; Wilson, 2007). Una estructura central del nexo entre tecnología y desarrollo se basa en el argumento de Schumpeter (1939) de que los ciclos de crecimiento económico de largo recorrido están impulsados por cambios en el paradigma sociotécnico que, efectivamente, transfiere la frontera de posibilidades de producción de un país a, un mayor valor agregado y a sectores económicos más productivos (Freeman \& Soete, 1997).

Sin embargo, Freeman (2001, p. 121) advirtió que "las burbujas, la euforia y los pánicos son fenómenos comunes durante la temprana difusión de las nuevas tecnologías, y las TIC no son diferentes a este respecto". También argumentó que, si bien las TIC estaban destinadas a hacer que los mercados funcionaran más eficientemente al reducir las fallas de información, esa información sobre los precios no necesariamente conducía a mejores decisiones de inversión, por ejemplo, y que por lo tanto sus impactos no deberían ser exagerados.

Acompañando las afirmaciones a menudo eufóricas y exageradas sobre el poder transformador de las TIC, hay un determinismo tecnológico que postula que la adopción de teléfonos móviles, ordenadores e Internet inevitablemente impulsará formas progresivas de modernización en regiones como África. Como argumentó Bimber (1990) los cálculos tecnodeterministas surgen de secuencias lógicas universales de desarrollo socioeconómico que acompañan la adopción de las nuevas tecnologías o, de manera paradójica, del potencial de interacción social. Las TIC pueden contribuir a las ganancias impulsadas por el conocimiento y la comunicación, pero también, a la innovación, empleo y prestación de servicios sociales, financieros y gubernamentales críticos. Sin embargo, como señaló Graham (1998, p. 180):

[... la misma noción de "impacto tecnológico", una característica central de los debates tecnológicos dominantes en los estudios urbanos y regionales, es problemática debido a sus implicaciones de causa simple, lineal y tecnológica y a sus efectos en la sociedad.

La difusión de las nuevas TIC está respaldada por la ideología, ya que se las representa como útiles para el empoderamiento individual y la interconexión acumulativa (inter) nacional, que cuando se combinan juntas constituyen ejes gemelos de la neoliberalización. Algunos van tan lejos como para argumentar que existe una ideología internacional de la tecnología de la información que combina "economías de libre mercado, políticas neoconservadoras y determinismo tecnológico” (Birdsall, 1996). Ya’u (2004, pp. 20-21) afirmó además que "las TIC han impulsado una nueva era del imperialismo en África: este nuevo imperialismo se caracteriza por el intento de creación de dependencia del conocimiento en los países recién recolonizados”.

Es una fórmula de soft power que no implica la ocupación física de los países y cuyos caminos están mediados por las vastas redes de TIC. Está señalizado por un mecanismo de control ejer-
Las TIC estaban destinadas a hacer los mercados más eficientes al reducir las fallas de información 
cido a través de la Organización Mundial del Comercio (OMC) que actúa en nombre de las potencias occidentales y sus empresas transnacionales. Está respaldado por una serie de medios de internalización ideológica que controlan el flujo de noticias, entretenimiento y literatura, así como por el espacio cultural en general. Este poder "imperial" se expresa a través del dominio del inglés en Internet, el estándar hegemónico del conocimiento occidental (supone el $52 \%$ del total de las páginas web mundiales ${ }^{8}$ ), y la capacidad del capital transnacional de mover flujos económicos instantáneos y de información en todo el mundo, así como de generar ganancias.

Como argumentó Castells (2003, p. 77), "la 'sociedad en red’ tiene y está imponiendo una forma particular de producción que da forma a las relaciones sociales a nivel mundial, a pesar de que las TIC se representan comúnmente como herramientas de empoderamiento personal”. Las TIC también han sido cómplices en la penetración más amplia y profunda del neoliberalismo en el Sur Global, que se manifiesta principalmente en formas intensificadas de competencia basadas en el libre mercado.

Según Dean (2012) la proliferación de información facilitada por las TIC se ha despolitizado. Otro ejemplo se relaciona con la financiarización de la economía global y la crisis económica mundial que comenzó en 2007-2008, de hecho, un resultado de la tergiversación de los valores de uso en los derivados financieros, cuya creación y comercialización se vieron facilitados por las TIC (Knox-Hayes, 2013). Esto no quiere decir que estas tecnologías no se utilicen con fines transgresores sino que, también, las utilizan los gobernantes para insertarse en las redes de poder existentes (Wasserman, 2011).

Zižek (1989) describió los objetos de ideología como aquellos sobre los que se proyectan propiedades que sirven funciones psicológicas para los sujetos. En este sentido, y como señalara Wilson (2014, p. 312):

[... el objeto sublime es un objeto material común que adquiere una fascinación peculiar por el sujeto, debido no a una esencia inherente, sino a su ubicación simbólica como un objeto que tanto oscurece y encarna el vacío de lo Real.

Considerado de esta manera, los teléfonos móviles, las computadoras e Internet son vistos implícitamente por muchos como objetos sublimes de la ideología. Mientras que la participación occidental en África - desde la esclavitud, hasta el colonialismo y el neoliberalismo- conllevó a una deplorable deshumanización, las TIC pueden representarse como un factor de desarrollo y modernidad y cumplen la función psicológica de expiar los pecados occidentales anteriores.

Los objetos sublimes son aquellos que tienen un profundo significado simbólico y sobrecargado para los sujetos que observan, y en el caso de las TIC, también los usan. Roy (2010) trazó el discurso sobre la "liberación" de las mujeres en el hemisferio sur a través de las microfinanzas. Roy (2010, p. 72) observó que, en un informe de microfinanzas del Banco Mundial (BM) la imagen de una mujer campesina usando un ábaco y una calculadora se presentaba como "un fetiche, un objeto mágico" que había superado el primitivismo para adoptar tecnologías modernas y calculadoras.

Imágenes similares son usadas frecuentemente por compañías telefónicas que muestran a guerreros masai con atuendos tradicionales usando móviles, pero en este caso, es posible que el receptor sea el objeto mágico que sirva como el símbolo de la modernidad. Tales fetichizaciones

8 Información a 2 de abril de 2018. Recuperado de https://w3techs.com/technologies/overview/content_language/all 
y objetivaciones dentro de las narrativas de Internet y desarrollo en África eluden muchas de las contradicciones que han acompañado a la revolución de los teléfonos móviles en África, particularmente en lo que se refiere a su potencial para transformar industrias y economías.

Si bien una amplia literatura ha examinado las formas en que las nuevas TIC pueden o podrían contribuir a la reducción de la pobreza, estas obras a menudo ignoran las formas en las que estas tecnologías se insertan en una economía política más amplia estructurada por el poder. Como describe Castells (2011), las TIC, la economía global de la información y la sociedad de redes se producen, integran y difunden a través de poderosas redes de actores, incluso cuando los actores menos poderosos derivan en una variedad de formas que son arrastradas a un espacio global de flujos.

\section{La virtualidad física: Facebook, Google, IBM y microsoft en África}

A finales de agosto de 2016 aparecía corriendo por las calles de Lagos, la capital económica de Nigeria. Es aparentemente tan accesible que no parece uno de ellos. Se asoma al mundo con aires despistados. Metro setenta y uno, ojos azules, pelo castaño, corto y rizado. Camisetas grises o negras, vaqueros y zapatillas de deportes. Sonríe mucho. Habla chino mandarín de forma fluida. Y como ocurrió con otras guerras, conquistar los corazones es la mejor forma de ganar una batalla. Una población libre se apresura a subir las últimas fotos y pensamientos en su invento. Mark Zukerberg es el creador de la red social más grande del mundo. A 31 de enero de 2018, Facebook tenía 2,13 mil millones de usuarios activos mensuales ${ }^{9}$, lo que hace de esta plataforma una mina en la que sus clientes potenciales se inscriben de forma gratuita y se exponen con información que hasta el momento ninguna encuesta telefónica ni agencia de inteligencia había logrado. Y al instante.

También parece preocuparse por la base de la estructura social como declaró a la cadena norteamericana CNN: "Hay tanta energía y potencial aquí [en Nigeria] que solo quiero caminar y conocer a la gente"10. Más allá de la crónica social que editaron algunos diarios internacionales -noticas presentadas con una dosis de exotismo- en su viaje a Nigeria se reunió con desarrolladores web, personas emprendedoras y agencias de comunicación. Un modelo que después extendió a un segundo país en su gira africana: Kenia.

Las repercusiones e implicaciones de que Zukerberg haya puesto el foco en el continente no son casuales. Y mucho menos que haya sido en Nigeria, el país más poblado de África con más de 180 millones de personas y con 16 millones de personas usuarias al mes conectados a Facebook, y en Kenia, con 4,5 millones de usuarios al mes.

La narrativa dominante de empresas como Google o Facebook es que pretenden conectar a millones de personas, pero vamos a tratar de trazar otras vinculaciones que nos ayudarán a entender esta dinámica que se insertaría en una guerra por el control de la información internacional y global. Un conflicto bélico que por uno de sus principales escenarios tiene al continente africano. Una carrera de armamentos digital que ya ha llegado. En esta competición no importa

9 Recuperado de https://zephoria.com/top-15-valuable-facebook-statistics/

10 Recuperado de http://money.cnn.com/2016/08/30/technology/facebook-mark-zuckerberg-africa-nigeria-lagos/ 
quién tiene la razón, sino quién aguanta el ritmo. Y, en este apocalipsis digital inminente, el claro favorito es Facebook.

\section{Cambridge Analytica en África a examen}

Apenas unos días después de que Facebook suspendiera a Cambridge Analytica por recopilar datos privados de 50 millones de perfiles para influir en las elecciones norteamericanas en las que se impuso Donald Trump ${ }^{11}$, los ejecutivos de esta consultora volvían a quedar expuestos. En un vídeo con cámara oculta publicado por Channel 4 News ${ }^{12}$ se descubría la hoja de ruta que habían seguido con la ayuda de Internet para envenenar a la democracia keniana y favorecer a Kenyatta en las elecciones de 2013 y 2017: "Hemos cambiado el nombre del partido dos veces, escrito el manifiesto, investigado, analizado y enviado mensajes... Creo que escribimos todos los discursos", explicaba Mark Turnbull, el director de la división política de Cambridge Analytica y de su compañía hermana SCL (Strategic Communication Laboratories Group $)^{13}$. Un documento que la propia consultora ha negado.

Un contexto que el keniano Moses Karanja, doctorando por la Universidad de Toronto y experto en la confluencia del binomio elecciones e Internet en África, explica de la siguiente manera:

Este es un fenómeno en crecimiento, el de los ciberguerreros. Cambridge Analytica puede haber ganado popularidad debido al odio extremo que crearon y a la atención de los medios debido a su vinculación en las elecciones estadounidenses y en el brexit. Sin embargo, creo que los verdaderos protagonistas son redes de personas pagadas por los Gobiernos para influir en los debates que se originan en las redes sociales ${ }^{14}$.

La explosión de nuevas aplicaciones digitales ha impulsado el acceso a la información, pero también ha fomentado una aversión a las perspectivas en profundidad. Algo que el keniano Leonard Wanyama, profesor de relaciones internacionales y especialista en desarrollo puntualiza:

Debido al fácil acceso a Internet, los kenianos de clase media son los sujetos más adecuados para la manipulación política. Quedan atrapados en el entretenimiento de un juego político; una condición que luego se sintetiza a las masas ya sea en la televisión o en la radio ${ }^{15}$.

\section{1. nigeria, el gigante africano en el punto de mira}

Pero el vídeo de Channel 4 News daba más de sí. En él, Turnbull mencionaba también el papel de Cambridge Analytica en las elecciones nigerianas de 2007 y 2015. Según informaba la agencia Reuters, un comité del Gobierno nigeriano está investigando las afirmaciones de que SCL Elections, consultora afiliada a Cambridge Analytica, organizó mítines en 2007 para

11 Recuperado de https://elpais.com/tag/caso_cambridge_analytica/a

12 Recuperado de https://www.youtube.com/watch?v=mpbeOCKZFfQ

13 Recuperado de https://qz.com/1233084/channel-4-news-films-cambridge-analytica-execs-saying-they-stagedkenya-uhuru-kenyatta-elections/

14 Entrevista realizada mediante correo electrónico.

15 Entrevista realizada mediante correo electrónico.

Este es un

fenómeno en crecimiento, el de los ciberguerreros 
disuadir a los partidarios de la oposición ${ }^{16}$ de votar en contra del Partido Democrático Popular (PDP) de Goodluck Jonathan. Informaciones que se sitúan en un precipicio ya que en 2019 hay convocadas elecciones presidenciales en este país, la primera economía regional y el principal exportador de petróleo africano. Un trofeo goloso, sin duda.

Ya en 2014, el Gobierno de Jonathan contrató los servicios de Levick ${ }^{17}$, una consultora con sede en Washington para asumir un concierto de 1,2 millones de dólares en el que se incluía la cláusula de ayudar al Gobierno a combatir al grupo terrorista Boko Haram. Un acuerdo que no fue casual por dos motivos: en primer lugar, unos meses después tendrían lugar los comicios en el país, y, en segundo lugar, esta consultora trabajaría para revalidar el mandato de Jonathan - cosa que no consiguió- tratando de desacreditar la exitosa campaña \#BringBackOurGirls ${ }^{18}$ que nació después del secuestro de las 276 niñas de Chibok ${ }^{19}$. Sin embargo, esta no es la primera vez que un líder nigeriano ha empleado este tipo de servicios. Durante la Guerra de Biafra entre 1966 y 1970, el Gobierno del país contrató a la empresa norteamericana de relaciones públicas Burson-Marsteller ${ }^{20}$ para contrarrestar los informes de genocidio de la guerra.

\subsection{El dorado africano para las consultoras internacionales}

Hay una idea que sobrevuela: la de África como laboratorio geopolítico durante las campañas electorales. Y quizás, uno de los casos más sonados de los últimos meses haya sido la humillación en Sudáfrica de una de las firmas de relaciones públicas más conocidas del Reino Unido, la consultora Bell Pottinger ${ }^{21}$. La compañía fue contratada por la familia Gupta, tres hermanos de la India que han construido un imperio multimillonario en la Nación del Arcoíris con su holding Oakbay. Los Gupta han sido ampliamente acusados de corrupción y de ejercer una influencia indebida sobre el expresidente sudafricano Jacob Zuma. Así que el papel de Bell Pottinger era el de desviar la atención mediática sobre los Gupta y Zuma, pero para ello, los británicos pervirtieron los métodos: llegaron a desencadenar tensiones raciales a través de una campaña masiva en redes sociales y sitios web con discursos llenos de odio ${ }^{22}$.

Otro ejemplo menos sonado es el de la consultora política Weber Shandwick, con sede en Nueva York, y el de la firma Cassidy \& Associates, ambas propiedades de la matriz InterPublic Group. Las dos rubricaron acuerdos con Egipto a finales de enero de 2017, ocho días después de que Trump asumiera el control de Estados Unidos ${ }^{23}$. Desde entonces se ha evidenciado las buenas relaciones de la Casa Blanca con el Gobierno de Abdel Fattah el-Sisi. En marzo de

16 Recuperado de https://www.reuters.com/article/us-facebook-cambridge-analytica-nigeria/nigerias-ex-presidentunaware-of-any-cambridge-analytica-involvement-in-elections-spokesman-idUSKCN1 HC2IN

17 Recuperado de https://www.prweek.com/article/1301011/levick-support-nigerian-government-searches-boko-haram

18 Recuperado de https:/verne.elpais.com/verne/2015/05/06/articulo/1430905506_382390.html

19 Recuperado de https://elpais.com/elpais/2015/01/24/planeta_futuro/1422105682_569189.html

20 Recuperado de http://www.infonews.com/nota/130856/el-plan-que-compro-la-dictadura-para-lavar

21 Recuperado de https://www.nytimes.com/2018/02/04/business/bell-pottinger-guptas-zuma-south-africa.html

22 Recuperado de https://www.theguardian.com/media/2017/sep/03/bell-pottinger-chief-quits-ahead-of-ruling-onrace-hate-campaign-claims

23 Recuperado de https://www.fara.gov/docs/6272-Exhibit-AB-20170128-3.pdf
Hay una idea que sobrevuela: la de África como laboratorio geopolítico durante las campañas electorales 
2018, el propio Trump reafirmaba esta asociación estratégica ${ }^{24}$ llamando a su homólogo egipcio que acaba de ser reelegido por un $97 \%$ de los votos en las elecciones presidenciales.

\section{El nuevo mercado de los gigantes tecnológicos}

Microsoft fue posiblemente la primera compañía global de tecnología en tomar un interés activo e importante en África. En 2013, la empresa comenzó su iniciativa 4Afrika ${ }^{25}$. Este programa de 75 millones de dólares fue diseñado para capacitar a miles de africanos ya fuera para sus propios negocios o para alguna de las 22 oficinas que la compañía tiene repartidas por el continente. De forma paralela, el programa se centró en conseguir dispositivos inteligentes y asequibles para millones de nuevos clientes.

Google ha implementado su proyecto $\operatorname{Loon}^{26}$ que, aunque no es exclusivo para África, sí que está pensado para conectar a las personas que habitan en lugares remotos o rurales, para llegar a las zonas con falta de cobertura, y para que una población determinada pueda volver a tener Internet después de una catástrofe. Además, Google tiene otras dos iniciativas notables en la región, Link $^{27}$ y Digify ${ }^{28}$.

Link ha instalado cables de fibra óptica en Kampala, la capital de Uganda, y también en Accra, la capital de Ghana, con un mayor despliegue. Digify es un compromiso importante para capacitar digitalmente a un millón de africanos. La compañía además se ha asociado con proveedores chinos como Huawei o Transsion ${ }^{29}$ para producir teléfonos inteligentes de presupuesto reducido de menos de 100 dólares que ya se venden en Nigeria, Egipto, Ghana, Costa de Marfil, Kenia o Marruecos.

IBM ha abierto nuevos centros de investigación, invertido en empresas locales, financiado un programa de habilidades informáticas de 60 millones de dólares, y ha creado nuevas iniciativas diseñadas para impulsar el uso de grandes volúmenes de datos, análisis y computación en la nube.

Y finalmente Facebook, que ha aparecido como el más agresivo de sus competidores con el proyecto "Internet.org" ${ }^{30}$. En 2016, la compañía trabajó en estrecha colaboración con más de una docena de operadores móviles de 17 países considerados emergentes en África y Asia. La iniciativa se ha implementado en Kenia, Sudáfrica, Tanzania, Senegal, Zambia, Ghana, Angola y Malaui. Uganda y Ruanda parecen ser los siguientes. Que comunidades aisladas y población empobrecida tengan acceso a la red de redes y a aplicaciones que mejoran la vida en diversos ámbitos como el de la salud, la energía o la agricultura no sería debatible. Pero sí que esta

\section{Microsoft fue posiblemente la primera compañía global de tecnología en tomar un interés activo e importante en África}

\footnotetext{
24 Recuperado de https://www.reuters.com/article/us-egypt-election-trump/trump-congratulates-egypts-sisi-in-callaffirms-strategic-partnership-idUSKCN1H91NG

25 https://www.microsoft.com/africa/4afrika/

26 https://x.company/intl/es-419_es/loon/

27 https://www.google.com/get/projectlink/

28 http://digifyafrica.com/

29 http://www.transsion.com/en

30 https://info.internet.org/es/
} 
iniciativa de Zukerberg haga una diferenciación entre sociedad de primera y segunda, como veremos ahora.

Para muchos, Internet es una comunidad global, pero la realidad es que dos tercios de la población mundial todavía no tienen acceso. Así que la pregunta es, ¿para qué quieren conectar a tanta gente?

En abril de 2014, Google adquirió Titan Aeroespacial, un fabricante de aviones no tripulados con sede en México a la que Facebook había cortejado con un contrato de 60 millones de dólares. Al parecer, Google subió la apuesta y acabó con la compra quitándole la empresa a Facebook. Pero la determinación de Zukerberg no desvaneció. El gigante de las redes sociales compró Ascenta, una empresa con sede en Reino Unido por 20 millones de dólares (unos 14,5 millones de euros). El hecho de que no haya leyes reguladoras de drones en muchas partes de África (Sudáfrica es una excepción) hace que la aventura de Facebook sea aún más plausible. A pesar de que la inversión de la infraestructura necesaria para la comunicación global va a ser extraordinariamente alta, la carretera parece que ya ha sido establecida: y se encuentra en el aire.

Tanto Facebook como Google, en su deseo de llegar a más personas online, explican su determinación en términos altruistas. Sin embargo, las empresas podrán ganar valiosos nuevos públicos para mostrar anuncios en un momento en el que la competencia en los países occidentales es intensa. Google ha afirmado que sus globos Loon podrían ser rentables si las empresas de telecomunicaciones los alquilan para ampliar sus redes ${ }^{31}$.

Esa mezcla de pensamiento y de caridad con visión empresarial ha supuesto un importante retroceso para una parte de la sociedad. El disfraz filantrópico del proyecto "Internet.org" de Zukerberg mantiene acuerdos con algunos operadores que ofrecen de forma gratuita acceso a Internet. Pero el acceso es a ciertos servicios, como el propio Facebook, el buscador de Google, Wikipedia, la BBC y otros servicios, entre ellos, algunos locales con los que han llegado a acuerdos.

Es decir, no se ofrece acceso gratuito a Internet, sino a "su Internet", el que ellos deciden, en una clara ruptura de la neutralidad de la red. En plena ola de protestas por esta discriminación, las empresas indias decidieron abandonar el proyecto en febrero de 2016 y, en mayo del mismo año, quejas similares estallaron en otros países, entre ellos Indonesia y Zimbabue ${ }^{32}$. "Internet.org” ni ofrecía Internet a sus usuarios ni era un “.org”, lo que aludiría a que se trata de una organización benéfica. Solo parecía ser un proxy encubierto para alcanzar a nuevos clientes con escasos recursos económicos.

\section{Conclusiones}

La determinación de los gigantes de la tecnología en África es implacable. Los países productores de más energía están teniendo un crecimiento proporcional en sus economías. En el mismo sentido, los expertos pronostican una escasez mundial de alimentos y de agua dulce que debería ser más preocupante que la falta de conectividad a Internet. Pero se ha convertido en una guerra entre Facebook y Google, Google contra Microsoft y así sucesivamente.

31 Recuperado de http://tecnologia.elpais.com/tecnologia/2017/02/17/actualidad/1487311146_799351.html

32 Recuperado de https:/www.theguardian.com/technology/2016/feb/08/india-facebook-free-basics-net-neutrality-row
Al no haber leyes reguladoras de drones en muchas partes de África hace que la aventura de Facebook sea aún más plausible 
Los pueblos de zonas remotas no deberían ser propiedad de nadie solo porque "Zuckerberg \& Cía." están buscando nuevos consumidores potenciales. Sin embargo, hay algunas ventajas que resultan de esta dura competencia, como las subvenciones en forma de planes de datos móviles a bajo costo o los teléfonos inteligentes a precios reducidos.

El mercado de productos y servicios de las empresas de tecnología se encuentra saturado en Europa y Norteamérica por lo que es comprensible que, enmarcado en la economía de mercado, los esfuerzos de publicidad se orienten a una población diferente a la del hemisferio norte. Por ejemplo, el mayor número de ventas de teléfonos inteligentes se están produciendo en la India o Filipinas. Nigeria, ese país en donde en agosto de 2016 se encontraba el creador de Facebook, es la segunda nación con la tasa más alta de suscripción al móvil; un margen relativamente pequeño y lejos del primer clasificado, la India. Pero ambos considerados mercados emergentes.

En esta línea, las consultoras políticas se han enfocado tradicionalmente en hacer tratos en las principales capitales mundiales como Londres, Nueva York, París y Washington. Sin embargo, lo que se ha vuelto más evidente en los últimos años es la presencia de estos sofisticados operativos de comunicación sobre la arena política africana como se está comprobando con Cambridge Analytica y algunos de sus servicios que han cruzado la línea del comportamiento ético aceptado. En este sentido, Fuchs argumenta que:

[... la crítica de la economía política de los gigantes de los medios globales es útil no solo para criticar el capital de los medios de comunicación, sino también como una manera de comprender diversas expresiones de las contradicciones subyacentes entre capital y trabajo y también como una forma de reflexionar y apoyar el potencial para la lucha de clases y alternativas al mundo de los medios capitalistas. (2017, p. 441)

Es difícil predecir qué calidad de acceso a Internet -y si aumentará todavía más la brecha digital- tendrán los próximos mil doscientos millones de usuarios africanos por los que estos gigantes de la tecnología están compitiendo. Lo cierto es que la información es poder y los datos son una especie de moneda. Los ganadores son los guardianes de esta valiosa información. Y Facebook está a punto de tomar ese papel.

\section{Referencias}

Abdi, J., Deane, J., \& BBC World Service Trust. (2008). The Kenyan 2007 elections and their aftermath: The role of media and communication. London: BBC World Service Trust.

Ba, A. (1999). Les Téléspectateurs africains à l'heure des satellites. Paris: L'Harmattan.

Banda, F. (2009). China in the African mediascape: a critical injection. Journal of African Media Studies, 1(3), 343-361. DOI: https://doi.org/10.1386/jams.1.3.343/1

Bimber, B. (1990). Karl Marx and the three faces of technological determinism. Social Studies of Science, 20(2), 333-351. DOI: https://doi.org/10.1177/030631290020002006

Birdsall, W. F. (1996). The Internet and the ideology of information technology. INET96 Proceedings 96. Recuperado de http://www.isoc.org/inet96/proceedings/e3/e3_2.htm

Castells, M. (2003). The networked society. En D. Held, \& A. G. McGrew (Eds.), The Global Transformations Reader: An Introduction to the Globalisation Debate. Oxford: Polity. 
Castells, M. (2011). A network theory of power. International Journal of Communication, (5), 773-787.

Dean, J. (2012). The limits to communication. Guernica: A Magazine of Art and Politics. Recuperado de http://www.guernicamag.com/features/the-limitsof-Communication/

Diamond, L. (2015). Facing Up to the Democratic Recession. Journal of Democracy, 26(1), 141-155. DOI: https://doi.org/10.1353/jod.2015.0009

Freeman, C. (2001). A hard landing for the 'New Economy'? Information technology and the United States national system of innovation. Structural Change and Economic Dynamics, (12), 115-139. DOI: https://doi.org/10.1016/S0954-349X(01)00017-0

Frère, M.-S. (2000). Presse et démocratie et Afrique francophone: Les Mots et les maux de la transition au Bénin et au Niger. Paris: Karthala.

Frère, M.-S. (2011). Elections and the media in post-conflict Africa: Votes and voices for peace? London: Zed Books.

Fuchs, C. (2017). Social media: A critical introduction. Los Angeles: Sage Publications.

Graham, S. (1998). The end of geography or the explosion of place? Conceptualizing space, place and information technology. Progress in Human Geography, 22(2), 165-185. DOI: https://doi.org/10.1191/030913298671334137

Karikari, K. (2004). Press Freedom in Africa. New Economy, 11(3), 184-186.

Karikari, K. (2010). African Media Breaks 'Culture of Silence'. African Renewal Online. Recuperado de https://doi.org/10.18356/aa8d9bfc-en

Knox-Hayes, J. (2013). The spatial and temporal dynamics of value in financialization: Analysis of the infrastructure of carbon markets. Geoforum, (50), 117-128.

Melber, H. (2016). The rise of Africa's Middle Class: Myths, realities and critical engagements. Uppsala: Nordiska Afrikainstitutet.

Nyamnjoh, F. B. (2005). Africa's Media: Democracy and the Politics of Belonging. London/Pretoria: Zed Books/Unisa Press.

Nye, D. (2006). Technology Matters: Questions to Live With. Cambridge, MA: MIT Press.

Oya, C., \& Santamaría, A. (2007). Economía política del desarrollo en África. Tres Cantos, Madrid: Akal.

Rogers, E. M. (1962). Diffusion of Innovations. New York: The Free Press.

Roy, A. (2010). Poverty Capital: Microfinance and the Making of Development. London: Routledge.

Ruiz-Giménez, I. (2012). Más allá de la barbarie y la codicia: Historia y politica en las guerras africanas. Barcelona: Bellaterra.

Ruiz-Cabrera, S. (2016). La ilusión del cuarto poder en Kenia: de los medios convencionales a las redes sociales. Chasqui, (129), 61-75. Recuperado de http://revistachasqui.org/index. php/chasqui/article/view/2502

Ruiz-Cabrera, S. (2017). La guerra (digital) por el control de la información en áfrica. Revista Pueblos, (71), 59-61. Recuperado de http://www.revistapueblos.org/?p=21395 
Ruttan, V. (2001). Technology, Growth, and Development. New York: Oxford University Press.

Schumpeter, J. A. (1939). Business Cycles: A Theoretical, Historical, and Statistical Analysis of the Capitalist Process. Volume I. New York: McGraw-Hill.

Shiffrin, A. (2017). In the Service of Power: Media Capture and the Threat to Democracy. Washington, DC: Center for International Media Assistance. Disponible en https://www.cima.ned. org/wp-content/uploads/2017/08/CIMA_MediaCaptureBook_F1.pdf

Tudesq, A.-J. (2002). L'Afrique parle, l'Afrique écoute: Les radios en Afrique subsaharienne. Paris: Karthala.

UNESCO. (2018). World Trends in Freedom of Expression and Media Development. Disponible en http://unesdoc.unesco.org/images/0026/002610/261065e.pdf

Vittin, T. (2002). Limpact des radios internationales en Afrique noire. En T. Mattelart (Ed.), La Mondialisation des médias contre la censure: Tiers monde et audiovisuel sans frontières (pp. 81-102). Bruxelles: De Boeck Supérieur.

Wasserman, H. (2011). Mobile phones, popular media, and everyday African democracy: transmissions and transgressions. Popular Communication: The International Journal of Media and Culture, 9(2), 146-158. DOI: https://doi.org/10.1080/15405702.2011.562097

Wilson, G. (2007). Beyond the dichotomies in technology for development. Geography Compass, 1(2), 119-135. DOI: https://doi.org/10.1111/j.1749-8198.2007.00005.x

Wilson, J. (2014). The shock of the real: the neoliberal neurosis in the life and times of Jeffrey Sachs. Antipode, 46(1), 301-321. DOI: https://doi.org/10.1111/anti.12058

Workneh, T. (2017). Sub-Saharan Africa. En B. Birkinbine, R. Gómez, \& J. Wasko (Eds.), Global Media Giants (pp. 287-310). New York: Routledge.

Ya'u, Y. Z. (2004). The new imperialism and Africa in the global electronic village. Review of African Political Economy, 31(99), 11-29. DOI: https://doi.org/10.1080/030562404200 0258397

Žižek, S. (1989). The Sublime Object of Ideology. London: Verso. 